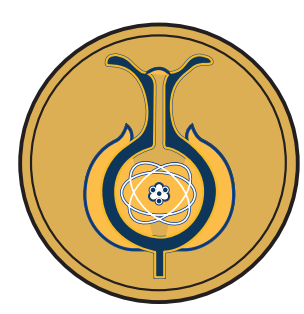

\title{
La demostración matemática: significado, tipos, funciones atribuidas y relevancia en el conocimiento profesional de los profesores de matemáticas
}

\author{
Mathematical proof: meaning, types, attributed functions, and relevance as \\ part of math teachers' professional knowledge \\ A demonstração matemática: significado, tipos, funções atribuídas e \\ relevância no conhecimento profissional dos professores de matemática
}

Cristian Alfaro-Carvajal

crisalfaro@correo.ugr.es

Universidad de Granada,

Granada, España.

Orcid: http://orcid.org/0000-0003-

2377-7181
Pablo Flores-Martínez

pflores@ugr.es

Universidad de Granada,

Granada, España.

Orcid: https://orcid.org/00000002-3292-6639

\section{Gabriela Valverde-Soto}

gabriela.valverde@ucr.ac.cr

Universidad de Costa Rica,

San José, Costa Rica.

Orcid: https://orcid.org/00000002-1319-9499

Recibido-Received: 30/jul/2018 • Corregido-Corrected: $15 / \mathrm{set} / 2018$.

Aceptado-Accepted: 25/set/2018 • Publicado-Published: 31/jul/2019.

\section{Resumen}

El objetivo de esta investigación es realizar un estudio teórico sobre el significado de la demostración matemática, considerando tres elementos centrales: el concepto, los tipos de demostraciones matemáticas, así como sus funciones. La indagación es de tipo cualitativo de carácter descriptivo. El método empleado para la recolección y el análisis de la información es el análisis conceptual. Se consideraron cuatro fuentes de datos: diccionarios, libros de texto, investigaciones previas y el programa de estudios de matemáticas del Ministerio de Educación Pública de Costa Rica. La técnica de recolección de los datos requeridos fue la revisión bibliográfica. Se determinó que el concepto de demostración tiene diversos sentidos, dependiendo del contexto en el que se ubique; que los tipos de demostraciones matemáticas pueden clasificarse en dos categorías, directas e indirectas, y que existen diferentes funciones atribuidas a las demostraciones matemáticas, las cuales cobran relevancia, dependiendo del ámbito en donde se consideren. Se cree que los tres elementos anteriores deben formar parte del conocimiento especializado del profesor de matemáticas, para que promuevan el sentido de la demostración en los estudiantes de la educación secundaria.

Palabras claves: demostración matemática; formación de profesores de matemáticas; análisis conceptual; análisis didáctico. 


\section{Abstract}

The objective of this paper is to conduct a theoretical study on the meaning of mathematical proof, taking into consideration three main elements: concept, types, and functions. This is a qualitative and descriptive research. The method used to collect and analyze information is the conceptual analysis. Four sources of information were considered: dictionaries, textbooks, previous research, and the mathematics curriculum of the Ministry of Public Education of Costa Rica. The technique for collecting the information was the bibliographic review. It was determined that proof has different meanings depending on the context; that the types of mathematical proof can be classified into two categories, direct and indirect; and that there are different functions attributed to mathematical proof which are relevant depending on the field in which they are considered. Consequently, the three previous elements should be part of math teachers' specialized knowledge to promote math proof in secondary education.

Keywords: mathematical proof; math teacher training; conceptual analysis; didactic analysis.

\section{Resumo}

0 objetivo desta pesquisa é realizar um estudo teórico do significado da demonstração matemática, considerando três elementos centrais: o conceito, os tipos de demonstrações matemáticas e suas funções. A indagação é do tipo qualitativa de caráter descritivo. 0 método utilizado para a coleta e análise de informações é a análise conceitual. Quatro fontes de dados foram consideradas: dicionários, livros de texto, pesquisas prévias e o programa de estudos de matemática do Ministério da Educação Pública da Costa Rica. A técnica para coleta dos dados solicitados foi a revisão bibliográfica. Determinou-se que o conceito de demonstração tem significados diferentes, dependendo do contexto em que está localizado; que os tipos de demonstrações matemáticas podem ser classificados em duas categorias, diretas e indiretas, e que existem diferentes funções atribuídas a demonstrações matemáticas, as quais se tornam importantes, dependendo da área considerada. Acredita-se que os três elementos acima devem fazer parte do conhecimento especializado do professor de matemática, para promover o sentido de demonstração em alunos do ensino médio.

Palavras-chave: demonstração matemática; formação de professores de matemática; análise conceitual; análise didática.

$\mathrm{S}$ egún Hanna y De Villiers (2011) la demostración es un tema relevante en la educación matemática, particularmente, en el currículo matemático de la secundaria. Existen dos posiciones sobre su enseñanza: como un contenido específico o como un estándar de proceso. En países como Francia, Alemania y Japón, la demostración es considerada un contenido explícito de enseñanza, de este modo, el programa de estudios establece lo que se debe apren- der y los libros de texto tienen capítulos dedicados a la enseñanza de la demostración. En otros países como Italia y los Estados Unidos, la demostración es considerada de una forma implícita e informal, particularmente, en los Estados Unidos es vista como un proceso que debe integrarse mediante el contenido matemático específico (Cabassut et al., 2011).

De acuerdo con las recomendaciones internacionales, como las del National 
Council of Teachers of Mathematics (NCTM, 2003), los programas de estudio de todos los niveles educativos deben favorecer en los estudiantes los procesos de razonamiento y demostración como elementos fundamentales de las matemáticas. Para ello, deben promoverse actividades tales como formular e investigar conjeturas, al igual que desarrollar y evaluar tanto argumentos matemáticos como demostraciones en las que se escojan y utilicen diferentes métodos de demostración y tipos de razonamientos.

En el caso del programa de estudios de matemática de la educación secundaria en Costa Rica, la demostración no es un contenido explícito, forma parte del proceso denominado razonar y argumentar, el cual corresponde a actividades mentales que involucran la deducción, la inducción, la comparación analítica, la generalización, las justificaciones, los ejemplos, contraejemplos y la demostración. La argumentación se debe promover paulatinamente, primero de forma verbal, luego por escrito $\mathrm{y}$, posteriormente, de modo simbólico. De igual manera, se deben introducir gradualmente las diferentes formas de razonamiento, hasta lograr procesos más formales y el uso de la deducción (MEP, 2012).

Con base en los planteamientos previos, es razonable considerar que los docentes de matemáticas deben tener un conocimiento profundo sobre el contenido de las demostraciones, es decir, deben poseer un saber específico sobre qué es una demostración matemática y por qué una demostración matemática es válida (Cabassut et al., 2011; Lin et al., 2011). Realizar demostraciones a sus estudiantes y promover que ellos las hagan es una tarea altamente demandante para el profesor de matemáticas, que exige un sólido conocimiento del contenido sobre las demostraciones matemáticas, pero también pueden tener influencia en su práctica profesional las concepciones que tenga sobre aquellas (Knuth, 2002).

A partir de lo anterior, se evidencia que es necesario que la demostración forme parte del conocimiento especializado del profesor de matemáticas; específicamente, en relación con tres elementos fundamentales: ¿qué es una demostración matemática?, ¿cómo se procede a demostrar en matemáticas? y ¿para qué sirven las demostraciones matemáticas? Las dos primeras preguntas están asociadas al concepto de demostración y a las formas de demostrar, la tercera tiene que ver con las funciones de la demostración.

\section{Marco teórico}

Según Rico (2001), una problemática en los procesos de investigación surge en el planteamiento de un marco teórico mal definido, en el cual se presenten términos o conceptos de manera errónea y con poca precisión. La multiplicidad de significados de los conceptos centrales propuestos en un marco teórico puede suponer una dificultad, si no se hace una precisión de ellos. Es por esto que "el análisis conceptual ofrece un método que permite al investigador convertir los conceptos en piezas teóricas precisas para el estudio que quiere llevar a cabo" (Rico, 2001, p. 185).

Se define el análisis conceptual como "un método para trabajar y profundizar los conceptos, una técnica de escrutinio para conseguir precisión y domino en su uso" (Rico y Fernández-Cano, 2013, p. 8). Según Rico (2001) y Rico y Fernández-Cano (2013), el análisis conceptual tiene las siguientes características: 
1. Es un método no empírico que trabaja con enunciados textuales: los datos que se consideran son descripciones, definiciones, listas extensivas, ejemplos de uso, la contraposición de textos con significados alternativos y formulaciones explícitas.

2. Está orientado por elementos tales como la naturalidad, la aplicabilidad, la complejidad y la simplicidad: realiza un cuidadoso examen de la diversidad de significados, las posibilidades de conexión entre los términos y los niveles objetivos (conceptos), subjetivos (creencias) e intersubjetivos (concepciones) de cada campo conceptual. Para una definición dada, considera el contexto en el que esta se inserta, usa ejemplos y contraejemplos en lugar de la definición explícita, utiliza analogías y términos evocativos en lugar de axiomas o cuantificadores y pruebas.

3. Se considera la historicidad y dinamicidad de los términos: hace una reflexión previa sobre lo que se desea investigar, de manera que se determine y caracterice los elementos fundamentales delimitadores del problema de estudio y las ideas, conceptos y teorías sobre las que se desea tratar. Además, procura descartar las inconsistencias que se puedan generar debidas a la poca precisión en el significado de los conceptos empleados.

4. Es un método esclarecedor para los educadores e investigadores en educación matemática: realiza una revisión profunda de los conceptos y nociones básicas sobre el conocimiento matemático, sus fundamentos e historia, su génesis y desarrollo, así como de los principios para su enseñanza e interpretación de su aprendizaje.

\section{Metodología}

Para realizar el análisis conceptual, las fuentes de información utilizadas fueron documentales. Se consideraron las siguientes: (1) diccionarios: etimológicos, de matemáticas, de educación matemática, de educación, de filosofía y de la lengua española; (2) libros de texto: de matemáticas, de educación matemática, de matemáticas en educación secundaria y de historia de las matemáticas; (3) investigaciones previas sobre la demostración matemática, y (4) el Programa de Estudios de Matemáticas de la educación secundaria en Costa Rica.

La técnica que se utilizó para recolectar los datos fue la revisión bibliográfica o de literatura. Acorde con Hernández-Sampieri, Fernández y Baptista (2014), esta revisión "implica detectar, consultar y obtener la bibliografía (referencias) y otros materiales que sean útiles para los propósitos del estudio, de donde se tiene que extraer y recopilar la información relevante y necesaria para enmarcar nuestro problema de investigación" (p. 61).

Una vez que fueron identificados y recopilados los trabajos de interés, se usó la ficha bibliográfica para incluir la información relevante de los documentos consultados: la referencia del documento, los datos sobre los autores, la naturaleza del documento, sus ideas principales, su ubicación y algunas observaciones pertinentes.

Para el análisis de la información recopilada, se hizo uso del análisis conceptual, el cual se centró en tres acercamientos a la noción demostrativa: (1) el concepto, considerando una revisión de las diferentes definiciones de la palabra demostración y otros términos asociados como argumentación, explicación, justificación, prueba y razonamiento; (2) los tipos, contemplando una revisión sobre las diferentes formas en las que 
se puede demostrar una proposición matemática (para ello, se consideró la diversidad de las proposiciones desde el punto de vista lógico), y (3) las funciones sobre las que se hizo una revisión de los diferentes roles que se pueden atribuir a las demostraciones matemáticas, debido a la variedad de significados de la palabra demostración, dependiendo del contexto en el que esta se ubique.

\section{Resultados}

En este apartado, se presenta una síntesis de los hallazgos del estudio teórico realizado. En cuanto al concepto de demostración, se discute el sentido informal y formal, la demostración clásica, la demostración en las matemáticas y en la educación matemática, además de la relación existente con los términos razonamiento, argumentación, explicación y prueba. Sobre los tipos de demostraciones en matemáticas, se consideran las demostraciones directas e indirectas, además de las distintas maneras en las que se debe proceder en función de las estructuras simbólicas de las proposiciones matemáticas involucradas. Finalmente, sobre las funciones atribuidas, se consideran los distintos roles de las demostraciones tanto en las matemáticas como en su enseñanza.

\section{El concepto de demostración matemática}

El término demostración puede utilizarse en un sentido informal en el que se haga referencia a sentimientos, habilidades o al funcionamiento de algo y, en uno formal, lógico o metodológico, de mayor tecnicismo, en el cual las demostraciones versan sobre proposiciones. Asimismo, es posible usarlo con diferentes enfoques, dependiendo del contexto o marco institucional, entendido como un punto de vista local que se caracteriza por el empleo de recursos propios y tanto por hábitos como por normas específicas. Algunos de estos contextos pueden ser la lógica y fundamentos de las matemáticas, la matemática profesional, la vida cotidiana, las ciencias experimentales y la enseñanza de las matemáticas elementales en todos sus niveles (Godino y Recio, 2001; Vega, 2012b).

En un sentido amplio, la demostración consiste en una prueba de algo, partiendo de verdades universales y evidentes, es un razonamiento convincente con el que se corrobora la veracidad de una proposición (Alvar, 1998; Comte-Sponville, 2005; Diccionario ilustrado Océano de la lengua española, 1994; Real Academia Española, 2006). En una línea más específica, Martí (2003) indica que la demostración es un razonamiento mediante el que se afirma la verdad de una proposición, aplicando las reglas de la lógica. Consta de tres partes: la tesis, entendida como lo que se quiere demostrar; un conjunto de proposiciones y una conclusión que se obtiene mediante razonamiento, la cual afirma la validez de la tesis.

\section{La demostración clásica}

Las demostraciones son pruebas deductivas formalizadas como las que se utilizan en la teoría de la prueba o las consideradas en la lógica de la demostrabilidad. Las pruebas que generalmente se han considerado como demostraciones en la metodología y la filosofía de la ciencia son las llamadas demostraciones clásicas, entendidas como una deducción que hace saber la necesidad racional de que algo sea el caso. Los paradigmas de este tipo de demostraciones son las deducciones matemáticas señaladas por 
las siglas QED (Quod eat demonstrandum). La demostración en su forma deductiva tiene interés también en contextos no matemáticos (Vega, 2012b).

Las demostraciones clásicas tienen características que permiten observar su relevancia en el discurso, la cognición y la argumentación. Tal es el reconocimiento social, en donde la validez de un argumento es reconocido o sancionado socialmente en la comunidad académica a la que pertenece; el cuerpo de conocimientos, el cual constituye una teoría deductiva en la que ninguna nueva demostración modificará su consistencia; la no admisión de contraprueba, que establece que toda proposición demostrada deductivamente en un cuerpo de conocimientos no admite una prueba deductiva para su negación; la claridad, la cual determina que demostrar una proposición es deducir que permite comprender que lo afirmado por tal proposición es efectivamente cierto (Vega, 2012b).

Para Vega (2012b), existen diferentes tipos de demostraciones clásicas correspondientes a los distintos modos de hacer saber: la demostración constatativa permite saber que una proposición es el caso, la demostración explicativa brinda la razón o la causa de por qué una proposición es el caso y la demostración fortísima reúne la fuerza de la demostración con su virtud explicativa. También existen diferencias entre la demostración directa y la indirecta, por reducción al absurdo. Las teorías matemáticas clásicas y los sistemas lógicos admiten ambos tipos de demostración; sin embargo, la lógica y la matemática intuicionistas o constructivistas no ven a la demostración indirecta con buenos ojos, particularmente, no admiten la validez del patrón deductivo que concluye que una proposición dada sea el caso, por el solo hecho de que su negación produce una contradicción.
Actualmente, las demostraciones clásicas, incluso en las matemáticas, son un extremo en el espectro general de las pruebas. En dicho espectro, existen pruebas por constatación mediante representaciones gráficas, como algunas demostraciones de geometría en las matemáticas de la antigua India que tenían un sustento empírico. Por ejemplo, la lógica nyaya generó una teoría de razonamiento fundamentada en la causalidad (Crespo, Farfán y Lezama, 2009). También, se pueden considerar los ensayos de pruebas y refutaciones como los dados por Lakatos (1978), quien considera que las matemáticas son falibles, pues los teoremas están sujetos al escrutinio constante y pueden ser rechazados mediante contraejemplos. De esta manera, las demostraciones, más que justificaciones, son una fuente de descubrimiento que permiten desarrollar conceptos y refinar conjeturas.

Otras demostraciones por tomar en cuenta son las pruebas deductivas hechas por computadora, como la prueba de Appel y Haken del teorema de los cuatro colores, que precisaba la verificación por computadora de 1482 configuraciones diferentes. Este tipo de pruebas no está sujeto a métodos axiomáticos y puede ser considerado como poco riguroso, lo que ha obligado a replantearse el significado y el rol de las demostraciones en las matemáticas (Kleiner, 1991). En este sentido, Tymoczko fue pionero al advertir los cambios suscitados en la concepción de las demostraciones matemáticas, a partir del teorema de los cuatro colores. Considera que las pruebas por computadora son evidencia suficiente a favor de una filosofía cuasi-empirista (Alcolea, 2007).

La diferencia fundamental entre las pruebas mencionadas anteriormente y las demostraciones clásicas es que la conclusión que se establece por estas últimas es 
un resultado que, en el cuerpo de conocimientos o la teoría deductiva en la que tiene lugar, tiene validez universal (Vega, 2012b).

\section{La demostración en Matemáticas}

El concepto de demostración matemática ha evolucionado históricamente. Lo que es una demostración y cuándo es válida es una construcción o comprensión relativa al contexto sociocultural que se considere. $\mathrm{La}$ mayor parte de las ciencias, incluyendo a las matemáticas, utiliza la inducción junto con la intuición para enunciar proposiciones. En las matemáticas, el razonamiento inductivo permite observar patrones en búsqueda de regularidades para la generalización y formulación de conjeturas. Estas conjeturas deben ser validadas o rechazadas, por lo tanto, se deben hacer demostraciones que requieren la deducción (Crespo y Farfán, 2005).

La demostración matemática es una práctica social de la comunidad matemática que tiene como principal objetivo validar el conocimiento matemático adquirido por la sociedad. En la actualidad, dicha demostración se rige por argumentaciones deductivas, sin embargo, históricamente esto no ha sido así. Existe evidencia de que los principios de la lógica clásica son construcciones socioculturales. En civilizaciones antiguas en Egipto, China y América precolombina, se usaron formas de argumentación diferentes a las utilizadas por los griegos. No obstante, en Occidente, la forma predominante de la argumentación matemática ha estado influenciada por la lógica de Aristóteles, el avance de la ciencia se ha basado en el razonamiento deductivo $\mathrm{y}$, por lo tanto, se ha asumido que dicha lógica es innata al ser humano (Crespo, Farfán y Lezama, 2010).
En las matemáticas interesa someter a un control lógico riguroso las hipótesis iniciales. Para ello, se escogen, mediante algún criterio de racionalidad, unos enunciados a los que se les da el nombre de axiomas o postulados. Una vez hecho esto, los únicos enunciados aceptables serán aquellos que se deduzcan de los axiomas, por medio de la inferencia lógica; dichos enunciados se llaman teoremas. Este tipo de deducción constituye el método axiomático. Un sistema axiomático tiene los siguientes elementos: (1) un idioma subyacente: una tabla de símbolos primitivos o alfabeto; (2) un repertorio de reglas de formación de fórmulas: unos serán términos indefinidos y otros serán términos formalmente definidos llamados definiciones; (3) las fórmulas primitivas del sistema: una lista de axiomas o postulados, que se suponen verdaderos, y (4) un sistema de lógica deductiva: un repertorio de reglas de inferencia. Los dos primeros elementos componen el lenguaje o la gramática y los otros dos, la lógica del sistema. La regla de inferencia establece que una fórmula, llamada conclusión, puede ser inferida de otras, llamadas premisas. (Garrido, 1991 y Roberts, 2010).

La demostración matemática es un proceso, un razonamiento, una serie de relaciones o una secuencia finita de fórmulas tales que cada una es un axioma o una consecuencia inmediata de algunas fórmulas precedentes, gracias a las reglas de inferencia. La fórmula final de la demostración se llama teorema o fórmula derivada. Se caracteriza por ser un género del discurso con una forma estrictamente codificada (Balacheff, 2000; Chambadal, 1976; Garrido, 1991; Lucena, 2005; Vera, 1960). Se basa en las definiciones, oraciones que dan significado a las palabras utilizadas en la demostración; los axiomas, proposiciones que obedecen a 
construcciones mentales, las cuales son necesarias para la organización del conocimiento, y los principios del razonamiento, las llamadas leyes del pensamiento que están implícitas en todos los campos del conocimiento de la humanidad, tales como la ley de identidad (plantea que la naturaleza esencial de las cosas es constante), la ley de contradicción (significa que una cosa no puede ser lo que es $\mathrm{y}$, al mismo tiempo, lo que no es) y la ley de exclusión del término medio (plantea que no hay nada intermedio entre las cosas contradictorias) (Patterson, 1950).

Realizar una demostración en matemáticas es un proceso cognitivo complejo que implica el uso de la intuición, la prueba, el error y el refinamiento para producir una demostración final de un teorema (Homero, 2007; Montoro, 2007). La mayoría de matemáticos considera que una demostración es más valiosa cuando favorece la comprensión; por esta razón, tales demostraciones se pueden ver más como entidades conceptuales, entendidas como una secuencia lógica de ideas matemáticas relacionadas, en las que el enfoque de derivación no es lo principal. De este modo, la calidad de una demostración matemática puede ser evaluada con criterios sintácticos, sin embargo, hacen falta otros. En ausencia de estos, generalmente se usan juicios de valor con un significado impreciso, tales como comprensible, ingenioso, explicativo, elegante, profundo, hermoso, entre otros. Dichos atributos van más allá de la corrección lógica de la demostración de un teorema, pero, en las matemáticas como en la educación matemática, esas propiedades cuasi estéticas, mal definidas, son muy importantes para el reconocimiento de una demostración como algo más que un certificado de la verdad (Hanna, 2014).

\section{La demostración en las mate- máticas escolares}

Según Stylianides (2007), la demostración en las matemáticas escolares es un argumento matemático que tiene las siguientes características: (1) un conjunto de menciones aceptadas: utiliza afirmaciones aceptadas como verdaderas por la comunidad del aula y que están disponibles para su uso, tales como las definiciones, los axiomas, los teoremas, entre otros; (2) los modos de argumentación: usa formas de razonamiento que son válidas para la comunidad del aula o que se ubican en el alcance conceptual de esta, como las reglas lógicas de inferencia, el uso de definiciones para derivar afirmaciones generales, la enumeración sistemática de todos los casos a los que se reduce una proposición cuando estos sean un número finito, la construcción de contraejemplos, el desarrollo de un razonamiento que muestra que se puede llegar a una contradicción, entre otros, y (3) los modos de representación de argumentos: la comunicación se lleva a cabo empleando formas de expresión apropiadas, conocidas y en el alcance conceptual de la comunidad de la clase, formas que incluyen el lenguaje oral, el uso de diagramas, las representaciones pictóricas, tabulares, entre otras.

La definición anterior excluye a los argumentos empíricos, es decir, aquellos basados en el uso de ejemplos que confirman y ofrecen una evidencia incompleta sobre la veracidad de una proposición matemática. La razón primordial de esta distinción es que no se considera pertinente llamar demostraciones a los argumentos matemáticamente no calificados; de esta manera, la demostración en la clase de matemáticas estaría en concordancia con las matemáticas como disciplina, donde los argumentos empíricos 
no se consideran demostraciones matemáticas. Lo anterior no significa que se deban devaluar las exploraciones empíricas para la identificación de patrones, la generación de conjeturas y obtener evidencias acerca de lo que se quiere demostrar (Stylianides, 2007).

Según Crespo, Farfán y Lezama (2010), en la clase de matemática existen formas de razonamiento que no están en concordancia con la conceptualización de la demostración matemática en un sistema axiomático, normalmente se generan por la transferencia a escenarios académicos de formas de argumentación utilizadas en contextos cotidianos no académicos. Se distinguen las argumentaciones abductivas, en las que se tienen como premisas una implicación, su consecuente y se concluye el antecedente; las argumentaciones inductivas, en las cuales se concluye la veracidad de una proposición a partir del examen de un número limitado de casos; las argumentaciones no monotónicas, en las que se pueden modificar los supuestos iniciales a partir de casos nuevos; las argumentaciones visuales que establecen conclusiones con base en diagramas; las argumentaciones a conocimiento cero, cuando se hace referencia a demostraciones que realmente no lo son, y las argumentaciones gestuales en las cuales se argumenta con gestos y ademanes.

\section{Términos asociados a la de- mostración: razonamiento, argumentación, explicación y prueba}

Elrazonamiento. Es un proceso esquemático que permite deducir una proposición llamada conclusión, a partir de una serie de proposiciones que se aceptan, denominadas premisas. El razonamiento se llama válido si la conclusión se deduce de las premisas mediante reglas o resultados aceptados en lógica, en caso contrario, se denomina inválido. La veracidad de una proposición que forma parte del razonamiento, desde el punto de vista real, depende de si lo expuesto en ella se ajusta o no a la experiencia o conocimiento de la realidad sobre la que predica. En general, la veracidad de una premisa se da como supuesto. De este modo, la validez de un razonamiento garantiza que, si se suponen verdaderas las premisas, se puede deducir la veracidad de la conclusión. Esta validez no garantiza la veracidad de las premisas ni de la conclusión (Valverde, 2012).

La argumentación. Para Vega (2012a), la palabra argumentar refiere a la forma de dar cuenta y razón de algo. alguien o ante alguien, con el objetivo de lograr su comprensión y aprobación. La argumentación es la acción de argumentar o el producto de ella, es una interacción discursiva e intencional presente en un conversatorio o en un texto que involucra a un agente, el cual brinda la razón de algo y unos destinatarios reales, potenciales o imaginarios. El argumento es una unidad discursiva básica en la argumentación, entendida como la actividad de argumentar o como un producto en un texto. El argumento se puede entender de diferentes maneras, dependiendo del enfoque de la argumentación, como se detalla:

1. El argumento en la argumentación discursiva: en las argumentaciones presentes en la práctica social regida por normas, convenciones, hábitos, entre otros elementos, el argumento es una acción o procedimiento cuyo objetivo es persuadir de manera racional a la persona a la que se dirige.

2. El argumento en la argumentación como producto textual de una 
interacción discursiva: el argumento es un conjunto de proposiciones dirigidas a mostrar que una de ellas está justificada por las restantes. Este tipo de argumento es el objeto favorito del análisis lógico de la argumentación y su propósito fundamental es justificar la conclusión con base en sus premisas. Su forma característica es la esquematización de sus tres componentes: premisas, conclusión y vínculo inferencial entre ellas, como se muestra a continuación.

Esquema 1: $P_{1} \cdots P_{n} \|-C$. Es decir, de las premisas $P_{1} \cdots P_{n}$ se sigue o se desprende como conclusión $C$.

Esquema 2: $C-\| P_{1} \cdots P_{n}$. Es decir, $C$ se sigue o se desprende como conclusión de las premisas $P_{1} \cdots P_{n}$.

Según Lo Cascio (1998), hay lugares canónicos para la argumentación y cada uno tiene su propio código: una conversación, una disputa, una discusión oral, un debate, una demostración matemática, etc. Por lo tanto, existen diferentes tipos de argumentaciones como la oral, la escrita, la coloquial, la formal, el texto argumentativo con la intención de demostrar y el texto argumentativo con la intención de persuadir. Además, diferencia a la argumentación en general de la argumentación demostrativa.

1. La argumentación demostrativa: parte de premisas indiscutibles y llega a conclusiones lógicas por procedimientos deductivos, los cuales son dictados por la lógica de la razón. Son razonamientos argumentativos universales centrados en lo racional y no tienden a convencer a un público en particular o a cambiar su opinión. El razonamiento filosófico y el matemático en general tienen carácter demostrativo, sin embargo, cuando intervienen elementos morales y éticos, se vuelve opinable, debido a que las premisas utilizadas requieren la aprobación del público.

2. La argumentación en general: no parte necesariamente de premisas indiscutibles para llegar a conclusiones lógicas, por lo tanto, no es siempre un razonamiento evidente e irrefutable. Los hablantes de una lengua en general tienden a justificar sus afirmaciones sin poderlas probar de manera formal. Estos intentos de justificación son las argumentaciones, por lo tanto, la argumentación es un complemento de la prueba formal y la teoría de la argumentación debe ser complementaria de la lógica formal, debe hacer referencia a los casos en los que se consideran valores $y$, por tanto, no es posible una verificación empírica o una prueba formal. La argumentación, cuando se basa en premisas y leyes subjetivas, genera un razonamiento que deviene opinable, es decir, que puede tener diversos resultados según el consenso de los interlocutores; de esta manera, su éxito no está asegurado como en la argumentación demostrativa.

Para Toulmin (1958), todos los tipos de argumentación son racionales y las normas de validez dependen del carácter de los problemas a los que hace referencia. Propone un modelo en el que considera seis categorías que pueden componer la argumentación: (1) los argumentos, los hechos, las pruebas, los datos o los argumentos que se tienen sobre un hecho determinado; (2) la opinión que corresponde a la tesis, la opinión o hipótesis 
avanzada, la pretensión inferida a partir de los datos; (3) la regla general, las garantías o reglas generales a partir de las que, si se tienen ciertos datos o argumentos, se pueden sostener y, por lo tanto, se justifican ciertas tesis u opiniones; (4) la fuente, el fundamento de las garantías o la fuente de las informaciones, es decir, datos ulteriores para sostener la tesis y que permiten garantizar las reglas generales o la verdad de los datos; (5) el calificador, el elemento que caracteriza, aunque relativizándolas, las tesis aducidas o los argumentos propuestos, y (6) la reser$v a$, las informaciones o datos que conducen a conclusiones o tesis hacia las que se está prevenido. Se trata de dudas y reservas sobre la validez $\mathrm{u}$ oportunidad de las tesis que ya han sido preanunciadas del hecho de que la tesis o conclusión se acompañe por un operador modal, es decir, por un calificador. El razonamiento es válido, si se ha seguido el procedimiento expuesto en el modelo y si la relación entre tesis y argumentos está justificada de forma conveniente.

Para Pedemonte (2007) la argumentación y la demostración en las matemáticas poseen características comunes: (1) son justificaciones racionales: tanto en la construcción de demostraciones matemáticas como en las argumentaciones se presentan justificaciones de los pasos desarrollados; (2) se realizan para convencer: ambas se desarrollan cuando se desea convencer de la veracidad de una afirmación, para ello se deben modificar las opiniones apelando a la racionalidad; (3) están dirigidas a una audiencia universal: las dos pretenden convencer a una audiencia que debe tener la capacidad de defender sus propias opiniones con respecto a la demostración o al argumento esbozado por el interlocutor, esta audiencia está compuesta por la comunidad matemática, el aula, el profesor, el mismo interlocutor, entre otros, y (4) pertenecen a un campo: las proposiciones que son objeto de demostración o argumentación matemática pertenecen a un campo teórico que delimita su validez (el álgebra, el análisis, la geometría, entre otros).

La explicación. Permite establecer y garantizar la validez de una proposición a un locutor. Está arraigada en sus conocimientos y en lo que para él son sus reglas personales de decisión de la verdad, es decir, su propia racionalidad. Cuando la explicación aparece en un discurso tiene como propósito hacer evidente al público la veracidad de una proposición que ya ha sido asumida por el locutor. No necesariamente se reduce a una secuencia deductiva y, en general, la base es el lenguaje natural (Balacheff, 2000).

La prueba. Refiere a una explicación que ha sido reconocida y aceptada socialmente por una comunidad. Sin embargo, esta aceptación no es definitiva, con el avance de los saberes en los que se apoya, puede evolucionar. Una prueba puede ser aceptada por una comunidad y rechazada por otra (Balacheff, 2000).

\section{Los tipos de demostraciones en matemáticas}

En general todas las proposiciones matemáticas que se desean demostrar tienen la forma $P \Rightarrow Q$ de manera explícita o implícita. Cuando no tenga la forma de manera explícita, se entiende que la proposición $P$ hace referencia a los axiomas, definiciones y teoremas que brindan información verídica sobre los objetos matemáticos a los que refiere la proposición $Q$ (Murillo, 2010).

Se pueden consideran dos tipos fundamentales de demostraciones matemáticas para proposiciones de la forma $P \Rightarrow Q$, las 
demostraciones directas y las demostraciones indirectas. Posteriormente, se discutirán otros tipos de demostraciones en función de estructuras simbólicas particulares, en las cuales las demostraciones directas o indirectas estarán presentes.

\section{Las demostraciones directas}

Estas demostraciones se basan en las reglas de inferencia silogismo hipotético $[(P \rightarrow Q) \wedge(Q \rightarrow R)] \rightarrow(P \rightarrow R)$ $\mathrm{y}$ el modus ponendo ponens $[P \wedge(P \rightarrow Q)] \rightarrow Q$. La proposición $P \Rightarrow Q$ será verdadera si es una tautología, es decir, si cada vez que la proposición $P$ es verdadera se sigue necesariamente que la proposición $Q$ es verdadera. En la demostración directa, se inicia suponiendo que $P$ es verdadera. Luego se debe construir una cadena de proposiciones $R_{1}, R_{2}, \cdots, R_{n}$ de forma tal que $P \Rightarrow R_{1}$ sea verdadera. Como $P$ es verdadera, entonces, por la regla de inferencia modus ponendo ponens, se sigue que $R_{1}$ es verdadera. Posteriormente, se debe garantizar que la proposición $R_{1} \Rightarrow R_{2}$ sea verdadera, como $R_{1}$ es verdadera por modus ponendo ponens se sigue que $R_{2}$ es verdadera. En general, la secuencia de implicaciones es la siguiente: $P \Rightarrow R_{1}, R_{1} \Rightarrow R_{2}, \cdots, R_{n} \Rightarrow Q$. Al final del proceso, se sigue necesariamente que la proposición $Q$ es verdadera (Bartle y Sherbert, 2004; Roberts, 2010).

Cuando se explicitan todas las proposiciones de la cadena y se indican todas las justificaciones para cada paso, la demostración se llama demostración formal. Una vez que se hace una redacción en prosa más simple en la que se omiten las justificaciones sobre las reglas de inferencia usadas y los presupuestos matemáticos que fundamentan cada paso de la demostración, esta recibe el nombre de demostración informal. Tal distinción es sobre la forma de presentación y no tiene que ver con el rigor, generalmente, se prefieren las demostraciones informales, pues son más simples de seguir (Bartle y Sherbert, 2004; Roberts, 2010).

\section{Las demostraciones indirectas}

Existen dos tipos de demostraciones indirectas para una proposición matemática de la forma $P \Rightarrow Q$, las demostraciones por contraposición y las demostraciones por reducción al absurdo (Bartle y Sherbert, 2004; Roberts, 2010).

1. Las demostraciones por contraposición: en lugar de demostrar la proposición $P \Rightarrow Q$ se demuestra la proposición contrapositiva $\neg Q \Rightarrow \neg P$. Este tipo de demostraciones se basan en la equivalencia lógica llamada contrapositiva $P \rightarrow Q \equiv \neg Q \rightarrow \neg P$. Para demostrar la proposición $\neg Q \Rightarrow \neg P$ se procede como en las demostraciones directas (Bartle y Sherbert, 2004; Roberts, 2010).

2. La demostración por reducción al absurdo: este tipo de demostraciones se basa en la equivalencia lógica 1lamada neutro, la cual establece que $P \vee F_{0} \equiv P$ en donde $F_{0}$ representa a cualquier contradicción. De esta manera, en lugar de demostrar la proposición $P \Rightarrow Q$ se demuestra la proposición equivalente La proposición $(P \Rightarrow Q) \vee F_{0}$ es equivalente a la proposición $(P \wedge \neg Q) \Rightarrow F_{0}$ que es la que en la práctica se demuestra. Para proceder con la demostración, se trabaja como si fuese una demostración directa, es decir, se supone 
que $P \wedge \neg Q$ es verdadera hasta deducir una proposición contradictoria que haría el papel de $F_{0}$ (Bartle y Sherbert, 2004; Roberts, 2010).

\section{Demostraciones conjunti- vas, disyuntivas, exhausti- vas, bicondicionales y con cuantificadores}

Como se mencionó anteriormente, las proposiciones matemáticas pueden presentar la estructura condicional $P \Rightarrow Q$ de manera explícita o implícita. En este apartado, se discute de modo breve la forma de proceder en proposiciones que involucran otros conectores lógicos. En cada uno de estos casos, se pueden hacer demostraciones directas o indirectas.

1. Demostraciones conjuntivas: para demostrar la proposición $P \wedge Q$ se deben demostrar por separado cada una de las proposiciones $P$ y $Q$ (Murillo, 2010).

2. Demostraciones disyuntivas: para demostrar la proposición $P \vee Q$ se puede utilizar la proposición equivalente $\neg P \Rightarrow Q$. Esto es posible en virtud de la equivalencia de implicación y disyunción que establece que $P \rightarrow Q \equiv \neg P \vee Q$ (Murillo, 2010).

3. Demostraciones exhaustivas o por casos: para demostrar la proposición $\left(P_{1} \vee P_{2} \vee \cdots P_{n}\right) \Rightarrow R$ se deben demostrar por separado cada una de las siguientes $n$ implicaciones $\quad P_{1} \Rightarrow R, P_{2} \Rightarrow R, \cdots, P_{n} \Rightarrow R$. Están basadas en la equivalencia lógica denominada exhaución $(P \vee Q) \rightarrow R \equiv[(P \rightarrow R) \wedge(Q \rightarrow R)]$ (Roberts, 2010).
4. Demostraciones bicondicionales: para demostrar la proposición $P \Leftrightarrow Q$ se deben demostrar por separado cada una de las siguientes dos implicaciones $P \Rightarrow Q$ y $Q \Rightarrow P$. Se basa en la definición del bicondicional $P \leftrightarrow Q \equiv[(P \rightarrow Q) \wedge(Q \rightarrow P)]$ (Murillo, 2010; Roberts, 2010).

5. Demostraciones con cuantificadores universales: Dada la proposición $\forall x \in U(P(x))$, en donde $P(x)$ es una frase abierta de variable $x$ en un dominio o universo de discurso $U$, se debe garantizar que todos los elementos de ese dominio hacen verdadera a la proposición que se obtiene al sustituirlos en la frase abierta. Se puede demostrar de manera directa, para ello se debe considerar un elemento arbitrario $x$ del universo $U$, y justificar que la proposición $P(x)$ es verdadera. También se puede demostrar de forma indirecta, específicamente por reducción al absurdo, al suponer cierta la proposición $\neg \forall x \in U(P(x))$, la cual es equivalente a suponer que $\exists x \in U(\neg P(x)), \quad$ es $\quad$ verdadera (Murillo, 2010; Roberts, 2010).

6. Demostraciones con cuantificadores existenciales: dada la proposición $\exists x \in U(P(x))$, en donde $P(x)$ es una frase abierta de variable $x$ en un dominio o universo de discurso $U$, se debe garantizar que existe al menos un elemento de ese dominio que satisface la frase abierta. Se puede demostrar de manera directa de dos formas: (1) mediante la llamada prueba constructiva, en la cual se exhibe o se explica cómo se construye un elemento $x$ del universo $U$, que 
verifica que la proposición $P(x)$ es verdadera, o (2) mediante la llamada prueba no constructiva, en la cual se garantiza la existencia de un elemento $x$ del universo $U$, que satisface la frase abierta utilizando otros teoremas (Murillo, 2010; Roberts, 2010). Cuando solamente un elemento del universo satisface una frase abierta, se tiene el llamado teorema de existencia y unicidad que simbólicamente se expresa $\exists ! x \in U(P(x))$. Esta proposición afirma dos cosas: que al menos un elemento del universo de discurso satisface la frase abierta y que solo ese elemento es capaz de satisfacerla. La proposición es equivalente a $(\exists x \in U(P(x)) \wedge \forall a, b \in U$ $[(P(a) \wedge P(b)) \Rightarrow a=b]) . \quad \mathrm{Se}$ puede demostrar directamente con cada una de las proposiciones por separado, como se indicó en la prueba conjuntiva, una parte demuestra la existencia y la otra la unicidad; cada una de estas partes es susceptible de demostrarse directa o indirectamente. Es posible demostrar de manera indirecta, por reducción al absurdo, suponiendo que la negación de toda la proposición dada es verdadera y a partir de esto se obtiene una contradicción (Roberts, 2010).

\section{Las funciones de las demos- traciones matemáticas}

Según Silva (2002) la demostración matemática tiene tres funciones:

\section{La función lógico-epistemológica: la demostración se entiende como una entidad objetiva existente en un}

espacio lógico, tiene una existencia matemática ideal, independientemente de que un agente real la conozca. Establece la veracidad de una afirmación matemática, al revelar las conexiones lógicas que garantizan la validez del enunciado, aunque no necesariamente logra la convicción, por ejemplo, en una demostración de $10^{25}$ pasos que es prácticamente imposible de seguir.

2. La función retórica: la demostración es una experiencia que permite generar una vivencia de convicción en un agente real, al posibilitar la comprensión de relaciones lógicas ideales. Es convincente sobre la veracidad de una afirmación, siempre y cuando se acepten los presupuestos de los que la demostración depende, esos que pueden ser injustificados, por lo tanto, podría ser lógicamente incorrecta $\mathrm{y}$, de este modo, no existe la garantía de la validez de la afirmación.

3. La función heurística: la demostración induce al conocimiento matemático, hace referencia al papel de la demostración según la perspectiva epistemológica falibilista de Popper (2000), la cual se representa en la filosofía de las matemáticas por la dialéctica de pruebas y refutaciones de Lakatos (1978). Esta función depende de la incorrección lógica de la demostración, lo que permite la formulación de contraejemplos que favorecen el progreso de las matemáticas.

Para Silva (2002), una demostración cumple la función lógico-epistemológica, si es correcta desde el punto de vista lógico, aunque no sea convincente. Puede satisfacer la función retórica si es convincente, aunque no necesariamente correcta y cumple 
la función heurística, si es lógicamente imperfecta. En consecuencia, pareciera complicado que una demostración matemática cumpla con las tres funciones señaladas, sin embargo, no es imposible. En efecto, una demostración lógicamente correcta puede constituir un desafío epistemológico, si su convicción induce una curiosidad intelectual en el sujeto, para pensar en variaciones de interés sobre las nociones implicadas que pudieran generar contraejemplos del resultado demostrado, lo que aporta a su función heurística. Normalmente, estas variaciones se logran mediante generalizaciones de los resultados a contextos más amplios en donde no hay garantía de validez.

Para que una demostración lógicamente correcta pueda ejercer la función retórica, debe estar acompañada de todos los pasos y cada uno de ellos debe ser comprendido. Es preciso que sea percibida por un ser humano, el cual debe ser convencido sobre sus bases racionales. Por tal razón, las demostraciones deben tener un número finito de pasos. Una demostración matemáticamente perfecta requiere ser lógicamente correcta, convincente para un ser humano con limitaciones cognitivas y estimulante desde el punto de vista heurístico (Silva, 2002).

Las demostraciones matemáticas han sido relegadas, casi de manera exclusiva, a la verificación de afirmaciones, lo que constituye una visión formalista que desvirtúa la naturaleza real de estas. En las últimas décadas, matemáticos y educadores matemáticos han diferido de la visión que resalta al razonamiento deductivo, el cual culmina en demostraciones formales, como el aspecto más relevante de las matemáticas. Las demostraciones pueden tener diferentes grados de validez formal y obtener el mismo nivel de aceptación (De Villiers, 1996; Hanna, 2002).
Para De Villiers (1996), las demostraciones matemáticas es posible que cuenten con las siguientes funciones:

1. La verificación: la demostración se considera como la máxima autoridad para asegurar la validez de una afirmación matemática. Se cree que detrás de cada teorema hay una secuencia de transformaciones lógicas para obtener la conclusión a partir de las hipótesis asumidas. Se categoriza esta visión como incorrecta, pues la demostración lógico-formal no es un garante de convicción.

2. La explicación: la demostración brinda las razones por las que una afirmación matemática es verdadera. Esta función es importante para comprender los motivos que hacen verdadero un resultado evidente, de manera intuitiva o por evidencia cuasi-empírica.

3. La sistematización: la demostración permite organizar varios resultados en un sistema deductivo de axiomas, definiciones y teoremas, lo que favorece la identificación de inconsistencias, verifica y simplifica teorías matemáticas y brinda una visión global sobre una temática que favorece sus aplicaciones en diferentes campos.

4. El descubrimiento: la demostración es un método de exploración, análisis, descubrimiento e inventiva, que permite descubrir nuevos resultados, los cuales serían difíciles de determinar de forma intuitiva o con procesos cuasi-empíricos. Un ejemplo de esta función es el descubrimiento de las geometrías no euclidianas, al modificar el postulado de las paralelas.

5. La comunicación: la demostración permite divulgar los resultados a los 
diferentes miembros de la comunidad científica: matemáticos, profesores, alumnos, entre otros. Debido a la complejidad del conocimiento matemático, se deben generar procesos de negociación subjetiva de significados de los temas involucrados, de manera que las argumentaciones sean aceptables. Esta función comunicativa expone las demostraciones a la sanción pública que permite refinar, simplificar, modificar y hasta refutar los resultados presentados.

En matemáticas, la convicción se puede lograr por formas alternativas a una demostración lógico-formal. La demostración es un argumento para validar una afirmación y puede asumir varias formas diferentes, siempre y cuando sea convincente. La aceptación de un teorema en matemáticas es un proceso social que depende más de la comprensión y el significado que de una demostración rigurosa. En general, los matemáticos aceptan un nuevo teorema cuando se tienen los siguientes factores: (1) la comprensión del teorema: son claros los conceptos incorporados en él, sus antecedentes lógicos y sus implicaciones; (2) la importancia: el teorema tiene implicaciones en al menos una rama de las matemáticas; (3) la compatibilidad: el teorema es consistente con los resultados matemáticos aceptados; (4) la reputación: el autor del teorema es reconocido como experto en la temática abordada, y (5) la convicción: se da un argumento matemático convincente, riguroso o no, de la validez del teorema (De Villiers, 1996; Hanna, 2002).

Cuando una prueba es válida solo en virtud de su forma, sin tener en cuenta el contenido, puede aportar poco a la comprensión del tema y puede resultar poco convincente. Los resultados matemáticos que se publican normalmente se presentan en forma de teoremas y demostraciones, aunque estas últimas no son evaluadas necesariamente por criterios de rigor. Cuando un matemático revisa una demostración pone su énfasis en las ideas matemáticas que están relacionadas en ella, la importancia, el objetivo que persigue y sus implicaciones. La adecuación lógico-formal en la que se presenta es algo secundario (Hanna, 2002).

Según De Villiers (1996), en la educación secundaria ha predominado la visión formalista de la demostración matemática, en donde la verificación de afirmaciones matemáticas es prácticamente la única función que se le atribuye. Considera que muchas de las dificultades que muestran los alumnos con las demostraciones están asociadas a la incapacidad de observar otras funciones que promuevan la comprensión de su significado, su propósito y utilidad.

Una manera de promover en los estudiantes la percepción de otras funciones de las demostraciones es mediante la formulación de conjeturas. La geometría euclídea es un área especialmente adecuada para este tipo de actividades, pues muchas veces se hace una introducción formal a los estudiantes sin permitirles una exploración experimental de las propiedades de las figuras geométricas. La introducción de algunos paquetes de software geométrico como Cabri-Geometry o Geometer's Sketchpad permite que los estudiantes realicen tales exploraciones al hacer construcciones geométricas complejas que pueden modificarse fácilmente. De este modo, se favorece la formulación de conjeturas y las argumentaciones experimentales que confirman resultados, pero que no explican necesariamente cómo tales resultados se derivan de otros que les son familiares; así se promueve 
la necesidad de hacer demostraciones matemáticas como una forma de explicación más que de validación (De Villiers, 1996).

Para una persona cuya preparación matemática es nula o parcial, puede parecer que el rigor y la forma de presentar una demostración es lo central en la práctica matemática, lo que puede inducir a que se conciba el aprendizaje de las matemáticas como un entrenamiento para crear estas formas. Lo fundamental es enseñar la importancia del razonamiento cuidadoso para la construcción de argumentos que puedan ser examinados y revisados con base en la claridad de ideas. La formalización es importante trabajarla, pero de manera secundaria (Hanna, 2002).

En la educación matemática, la principal función de la demostración es la explicación. Por lo tanto, la demostración en la clase de matemática no debe presentarse como un ritual que refleje de manera vaga e imprecisa la práctica matemática, por el contrario, debe abordarse como una actividad educativa con significado. Una demostración puede ejercer la función explicativa de diferentes maneras, dependiendo del grado y el contexto en el que se realiza la enseñanza: la realización de un cálculo, una prueba preformal, una prueba informal o una prueba estrictamente rigurosa. El elemento en común en todos los niveles y contextos es que los estudiantes están aprendiendo matemáticas y que se les dan resultados que ellos saben que son verdaderos. El reto es hacerles comprender por qué son verdaderos (Hanna, 1995).

Para que una demostración logre la función explicativa, el profesor de matemáticas debe lograr que los estudiantes comprendan los conceptos utilizados, que conozcan los patrones de argumentación y los términos involucrados, como suposición, conjetura, ejemplo, contraejemplo, refutación y generalización. Debe estructurar y presentar la demostración para que sea clara y convincente. En la medida que los estudiantes aprendan modos de pensamiento lógico, adquirirán la capacidad y la confianza para evaluar y construir una demostración matemática. Muchas demostraciones se pueden reestructurar para que se utilicen en la clase de matemáticas; no obstante, podrían presentar diferencias sustanciales en cuanto a su poder explicativo. Es posible distinguir entre las demostraciones que prueban y las demostraciones que explican. Las primeras justifican que un teorema es verdadero, las segundas logran lo mismo, pero la evidencia que se presenta se deriva del fenómeno al cual refiere tal teorema (Hanna, 1995).

Un ejemplo de lo anterior lo propone Hanna (1995), al considerar el teorema que afirma que la suma de los primeros $n$ números naturales es $\frac{n(n+1)}{2}$. Este teorema se puede demostrar mediante el principio de la inducción matemática, sin embargo, tiene poco valor explicativo porque no le indica a los estudiantes el porqué es verdadero. Una demostración que explica podría mostrar la razón de la validez del teorema, al utilizar dos representaciones de la suma, como se muestra a continuación:

$$
\begin{aligned}
& S=1+2+3+\cdots+n \\
& S=n+(n-1)+(n-2)+\cdots+1 \\
& 2 S=(n+1)+(n+1)+\cdots+(n+1) \\
& 2 S=n(n+1) \\
& S=\frac{n(n+1)}{2} .
\end{aligned}
$$




\section{Conclusiones}

La palabra demostración tiene diversos significados dependiendo del contexto en el que se le considere. En contextos informales, puede hacer alusión a la evidencia de sentimientos, habilidades y al funcionamiento de algún objeto; de manera más formal, refiere a garantizar la validez de proposiciones. No obstante, aun en contextos formales existen diferencias en cuanto a lo que es una demostración, en efecto, hay pruebas por constatación que utilizan representaciones gráficas, procedimientos empíricos, pruebas por computadora y las llamadas demostraciones clásicas que son pruebas deductivas formalizadas.

La demostración matemática no es ajena a la variedad de significados y a la evolución histórica. En consecuencia, qué es una demostración matemática y cuándo es válida son elementos fuertemente vinculados al contexto sociocultural. En lo que parece existir consenso es en que su objetivo fundamental es el de validar el conocimiento matemático generado por la sociedad, las diferencias radican en la manera de hacerlo. Por ejemplo, en civilizaciones antiguas en India, Egipto, China y América precolombina, las formas argumentativas empleadas diferían de las utilizadas por los griegos. En el caso de Occidente, la demostración matemática ha sido influenciada por la lógica aristotélica, es decir, por el razonamiento deductivo, y se ha tomado como postulado que dicha lógica es natural en el ser humano.

Formalmente, se puede entender la demostración matemática como un proceso, un razonamiento o una secuencia de fórmulas, de manera que cada una de ellas es un axioma o una consecuencia de fórmulas precedentes, mediante las reglas de inferencia.
Bajo este enfoque, se pueden considerar como un discurso estrictamente codificado. Una demostración matemática no establece un hecho $A$, sino una proposición del tipo $s i$ $B$ entonces $A$, lo que indica que los teoremas matemáticos son afirmaciones condicionales que dependen de los elementos del sistema axiomático de la teoría en la que están involucrados. Para demostrar, es necesario responder dos cuestiones básicas: ¿qué significa que una afirmación matemática sea verdadera? y ¿qué debería hacerse para demostrar que una afirmación matemática es cierta? Las respuestas a ambas preguntas dependen directamente de las reglas de inferencia de la lógica de predicados y de la estructura sintáctica de las definiciones involucradas, por tal razón, se hace relevante el conocimiento sobre los tipos de demostraciones matemáticas: directas e indirectas.

Otro elemento fundamental de las demostraciones matemáticas es el grado de convicción. Aunque las demostraciones tienen el rol de validar el conocimiento matemático, también es importante su función explicativa, mediante la que se brinda las razones por las que una afirmación matemática es verdadera.

En la educación secundaria de Costa Rica, el papel del profesor de matemática es fundamental para promover que los estudiantes se familiaricen con el sentido de la demostración matemática. Debe realizar demostraciones de algunos teoremas y solicitar a los estudiantes que ejecuten demostraciones sobre algunos resultados matemáticos. La demostración es considerada una fase formal de la argumentación y tiene un papel relevante en la formulación de conjeturas. Una vez que los estudiantes han formulado una conjetura, se deben trabajar tres etapas: en la primera, es preciso hacer la verificación en casos particulares; 
en una segunda, los estudiantes deben proponer un argumento que justifique la validez de la conjetura, y, finalmente, en una tercera etapa deben realizar la demostración (MEP, 2012).

Lo anterior sugiere que la demostración matemática debe formar parte del conocimiento especializado del profesor. Para ello, debe tener un dominio sobre el concepto de demostración y las maneras de entenderse en función del contexto en que se considere: la vida cotidiana, las matemáticas y la educación matemática. Debe conocer las formas de proceder para realizar demostraciones matemáticas, lo cual requiere un conocimiento adecuado de las reglas de inferencia lógicas, de la estructura sintáctica de las afirmaciones matemáticas y de los tipos de demostraciones. Además, es importante el conocimiento sobre los roles que tienen en las matemáticas, en los cuales destaca la función explicativa, pues favorece la comprensión sobre por qué una afirmación matemática es verdadera.

Algunas investigaciones han evidenciado que los profesores de matemáticas identifican, de manera correcta, un argumento válido, pero también aceptan argumentos inválidos como demostraciones. Por otra parte, los criterios utilizados por los profesores para evaluar un argumento difieren mucho entre sí, aunque existen algunos elementos comunes como los esquemas simbólicos o rituales, la forma del argumento, las manipulaciones algebraicas utilizadas, entre otros. Sobre las funciones de la demostración, algunas investigaciones muestran que los profesores se inclinan por la verificación de resultados como la principal función en detrimento de la función explicativa y la de promover la comprensión. Además, los profesores encuentran que un argumento es convincente por factores tales como el uso de ejemplos concretos, la referencia visual y no necesariamente su validez lógico-matemática (Cabassut et al., 2011).

Con base en lo anterior, es necesario que el profesor de matemática conozca la variedad de significados de la palabra demostración, pero es fundamental que tenga claridad sobre lo que es una demostración matemática, sobre cómo se demuestra una proposición matemática y el para qué realizar demostraciones matemáticas. Asimismo, es importante su conocimiento sobre procesos asociados como el razonamiento, la explicación y la argumentación, de forma que pueda diferenciarlos y encontrar elementos de convergencia.

\section{Reconocimientos}

Este trabajo se enmarca en las actividades del Programa de Doctorado en Ciencias de la Educación, específicamente, en el Proyecto «Conocimiento Didáctico del Profesor y Aprendizaje de Conceptos Matemáticos Escolares» (EDU2015-70565-P) del Plan Nacional de I+D+I (MICIN) y del Plan Andaluz de Investigación, Desarrollo e Innovación (Grupo FQM-193, Didáctica de la Matemática. Pensamiento Numérico).

\section{Referencias}

Alcolea, J. (2007). Razonamientos no rigurosos y demostraciones asistidas por ordenador. Contrastes. Revista Internacional de Filosofía, 12. doi: http://dx.doi.org/10.24310/Contrastescontrastes.v12i0.1432

Alvar, M. (1998). Diccionario ideológico de la lengua española. España, Barcelona: Bibliograf, S. A.

Balacheff, N. (2000). Procesos de prueba en los alumnos de matemáticas. Una empresa docente. Colombia, Bogotá: Editorial de la Universidad de los Andes. Recuperado de https://hal.archives-ouvertes.fr/hal-00520133/document 
Bartle, R. y Sherbert, D. (2004). Introducción al análisis matemático de una variable. México, Ciudad de México: Editorial Limusa, S. A. de C. V.

Cabassut, R.; Conner, A.; İşçimen, F. A.; Furinghetti, F.; Jahnke, H. N. y Morselli, F. (2011). Conceptions of proof-In research and teaching. En Proof and proving in mathematics education (pp. 169-190). Dordrecht: Springer. Doi: https://doi.org/10.1007/978-94-007-2129-6_7

Chambadal, L. (1976). Diccionario de las matemáticas modernas. Francia, París: Larousse.

Comte-Sponville, A. (2005). Diccionario filosófico. España, Barcelona: Ediciones Paidós Ibérica, S. A.

Crespo, C. y Farfán, R. (2005). Una visión socioepistemológica de las argumentaciones en el aula. El caso de las demostraciones por reducción al absurdo. Revista Latinoamericana de Investigación en Matemática Educativa, 8(3), 287-317. Recuperado de http://www.redalyc. org/pdf/335/33508304.pdf

Crespo, C.; Farfán, R. y Lezama, J. (2009). Algunas características de las argumentaciones y la matemática en escenarios sin influencia aristotélica. Revista latinoamericana de investigación en matemática educativa, 12(1), 29-66.

Crespo, C.; Farfán, R. y Lezama, J. (2010). Argumentaciones y demostraciones: una visión de la influencia de los escenarios socioculturales. Revista latinoamericana de investigación en matemática educativa, 13(3), 283-306. Recuperado de http://www.scielo. org. $\mathrm{mx} / \mathrm{scielo} . \mathrm{php}$ ?script=sci_arttext\&pi$\mathrm{d}=\mathrm{S} 1665-24362010000300003$

De Villiers, M. (1996). Future of secondary school geometry. Recuperado de http://mzone. mweb.co.za/residents/profmd/future.pdf

Diccionario ilustrado Océano de la lengua española. (1994). España, Barcelona: OCEANO.

Garrido, M. (1991). Lógica simbólica. España, Madrid: Editorial Tecnos.

Godino, J. D. y Recio, Á. M. (2001). Significados institucionales de la demostración. Implicaciones para la educación matemática. Enseñanza de las ciencias: revista de investigación y experiencias didácticas, 19(3), 405-414. Recuperado de http://www.raco.cat/index.php/ Ensenanza/article/viewFile/21763/21597

Hanna, G. (1995). Challenges to the importance of proof. For the Learning of mathematics, 15(3), 42-49. Doi: https://doi. org/10.1007/0-306-47203-1_4
Hanna, G. (2002). Mathematical proof. En D. Tall (Ed.), Advanced mathematical thinking (pp. 54-61). Dordrecht: Springer. Doi https://doi. org/10.1007/978-94-007-2129-6 1

Hanna, G. (2014). The width of a proof. $P N A, 9(1)$, 29-39. Recuperado de http://digibug.ugr. es/bitstream/10481/33232/1/Hanna2014PNA9(1)Thewidth.pdf

Hanna, G. y De Villiers, M. (2011). Aspects of proof in mathematics education. En G. Hanna y M. De Villiers (Eds.), Proof and Proving in Mathematics Education (pp. 1-10). Dordrecht: Springer. Recuperado de https://link.springer. com/chapter/10.1007/978-94-007-2129-6_1

Hernández-Sampieri, R.; Fernández, C. y Baptista, M. (2014). Metodología de la Investigación (6.a edición). México: McGRAW-HILL.

Homero, Á. (2007). Esquemas de argumentación en profesores de matemáticas del bachillerato. Educación Matemática, 19 (1), 63-98. Recuperado de http://www.redalyc.org/articulo. oa? id=40519104

Kleiner, I. (1991). Rigor and proof in mathematics: A historical perspective. Mathematics Magazine, 64(5), 291-314. Doi https://doi.org/10.1 080/0025570X.1991.11977625

Knuth, E. J. (2002). Secondary school mathematics teachers' conceptions of proof. Journal for research in mathematics education, 33(5), 379-405. Recuperado de https:/www.jstor. org/stable/pdf/4149959.pdf?refreqid=excelsior $\% 3$ A3130897e 8847 df8383552edfbe$28 \mathrm{c} 44 \mathrm{e}$

Lakatos, I. (1978). Pruebas y refutaciones. La lógica del descubrimiento matemático. Madrid: Alianza Universidad.

Lin, F. L.; Yang, K. L.; Lo, J. J.; Tsamir, P.; Tirosh, D. y Stylianides, G. (2011). Teachers' professional learning of teaching proof and proving. En G. Hanna y M. De Villiers (Eds.), Proof and proving in mathematics education (pp. 327-346). Dordrecht: Springer. Doi https:// doi.org/10.1007/978-94-007-2129-6 14

Lo Cascio, V. (1998). Gramática de la argumentación: estrategias y estructuras. España, Madrid: Alianza Editorial, S. A.

Lucena, N. (2005). Diccionario esencial de Matemáticas. España, Barcelona: SPES Editorial, S. L.

Martí, I. (2003). Diccionario enciclopédico de educación. España, Barcelona: Grupo Editorial Ceac, S. A. 
Ministerio de Educación Pública. (2012). Programas de estudio de matemáticas I, II y III ciclos de la educación general básica y ciclo diversificado. Costa Rica, San José: autor Recuperado de https://mep.go.cr/sites/default/files/programadeestudio/programas/matematica.pdf

Montoro, V. (2007). Concepciones de estudiantes de profesorado acerca del aprendizaje de la demostración. Revista electrónica de investigación en educación en ciencias, 2(1), 101-121. Recuperado de http://www.scielo.org.ar/scielo.php?pid $=$ S1850-66662007000100006\&script=sci_arttext\&tlng $=\mathrm{pt}$

Murillo, M. (2010). Introducción a la matemática discreta. Costa Rica, Cartago: Editorial Tecnológica de Costa Rica.

National Council of Teachers of Mathematics (NCTM). (2003). Principios y estándares para la educación matemática. Sevilla: Thales.

Patterson, C. (1950). Los principios del pensamiento correcto: lógica. Argentina, Buenos Aires: Editorial Americalee.

Pedemonte, B. (2007). How can the relationship between argumentation and proof be analysed? Educational Studies in Mathematics, 66(1), 23-41. Doi https://doi.org/10.1007/ s10649-006-9057-x

Popper, K. (2000). La sociedad abierta y sus enemigos. Barcelona: Paidós.

Real Academia Española. (2006). Diccionario esencial de la lengua española. España, Madrid: Espasa Calpe. Recuperado de http://www.rae.es/

Rico, L. (2001). Análisis conceptual e investigación en Didáctica de la Matemática. España: Universidad de Granada. Recuperado de http://funes. uniandes.edu.co/523/1/RicoL01-2593.PDF
Rico, L. y Fernández-Cano, A. (2013). Análisis didáctico y metodología de investigación. En L. Rico., J. Lupiañez. y M. Molina (Eds.), Análisis Didáctico en Educación Matemática: metodología de investigación, formación de profesores e innovación curricular (pp.1-22). Granada: Comares, S. L.

Roberts, C. (2010). Introduction to mathematical proofs: a transition. USA, New York: Chapman y Hall/CRC.

Silva, J. (2002). Demonstração Matemática da Perspectiva da Lógica Matemática. BOLEMA: Boletim de Educação Matemática, 15, 68-78.

Stylianides, A. J. (2007). Proof and proving in school mathematics. Journal for research in Mathematics Education, 38(3), 289-321. Recuperado de http:// www.jstor.org/stable/30034869?seq=1\#page_ scan_tab_contents

Toulmin, S. (1958). The use of arguments. Cambridge: University Press.

Valverde, L. (2012). Introducción al razonamiento lógico matemático. Costa Rica, San José: Editorial UCR.

Vega, L. (2012a). Compendio de lógica, argumentación y retórica. En L. Vega. y P. Olmos (Eds.), Argumentación (pp. 66-74). Madrid: Editorial Trotta, S. A.

Vega, L. (2012b). Compendio de lógica, argumentación y retórica. En L. Vega. y P. Olmos (Eds.), Demostración (pp. 182-184). Madrid: Editorial Trotta, S. A.

Vera, F. (1960). Matemática: lexicón kapelusz. Argentina, Buenos Aires: Editorial Kapeluz.

\section{(c) (i) $(9)$}

La demostración matemática: significado, tipos, funciones atribuidas y relevancia en el conocimiento profesional de los profesores de matemáticas (Cristian Alfaro Carvajal y otros) por Revista Uniciencia se encuentra bajo una Licencia CreativeCommons Atribución-No-

Comercial-SinDerivadas 3.0 Unported. 